ISSN 1991-8631

Original Paper

http://indexmedicus.afro.who.int

\title{
Émissions de dioxyde de carbone et de méthane des termitières de Macrotermes bellicosus au Burkina Faso
}

\author{
B. Joseph SAWADOGO ${ }^{1^{*}}$, Dayéri DIANOU ${ }^{2}$ et S. Alfred TRAORE ${ }^{1}$ \\ ${ }^{1}$ Centre de Recherche en Sciences Biologiques Alimentaires Nutritionnelles (CRSBAN), 03 BP 7131 \\ Ouagadougou 03, Burkina Faso. \\ ${ }^{2}$ Centre National de la Recherche Scientifique et Technologique (CNRST), 03 BP 7192 Ouagadougou 03, \\ Burkina Faso. \\ "Auteur correspondant, E-mail: josephsawadogo@yahoo.fr
}

\section{RESUME}

Cette étude a pour but d'évaluer les émissions de gaz carbonique $\left(\mathrm{CO}_{2}\right)$ et de méthane $\left(\mathrm{CH}_{4}\right)$ à partir des termitières de Macrotermes bellicosus appartenant à la famille des Termitidae qui occupe plus de 2/3 des sols tropicaux. Deux différentes termitières $\left(T_{1}\right.$ et $\left.T_{2}\right)$ ont fait l'objet de cette étude. Des échantillons gazeux ont été prélevés des termitières puis analysés par chromatographie en phase gazeuse. Des concentrations variables de $\mathrm{CO}_{2}$ ont été enregistrées de chaque termitière durant la nuit (22368 et 12803 ppm respectivement pour $\mathrm{T}_{1}$ et $\mathrm{T}_{2}$ ) et le jour (21397 et $9985 \mathrm{ppm}$ respectivement pour $\mathrm{T}_{1}$ et $\mathrm{T}_{2}$ ). Selon l'activité des termites, l'architecture des termitières et le gradient de température entre l'intérieur et l'extérieur des termitières, des mécanismes de ventilation sont élaborés par ces termites pour diffuser les gaz. Des traces de $\mathrm{CH}_{4}(<0,5 \mathrm{ppm}$ $\mathrm{CH}_{4}$ / jour) ont été aussi détectées tout au long de l'étude quelle que soit la température des termitières. Par contre, la transition saisonnière (sèche à pluvieuse) a marqué une très légère augmentation d'émission de $\mathrm{CH}_{4}$ $\left(0,5 \mathrm{ppm} \mathrm{CH}_{4} /\right.$ jour$)$. En plus de ces facteurs, bien d'autres paramètres sont à intégrer pour une étude ultérieure plus complète.

(C) 2012 International Formulae Group. All rights reserved.

Mots clés: Gaz à effet de serre, termites, structure des termitières, température, cycle journalier.

\section{INTRODUCTION}

Le rapport mondial sur le développement humain stipule que, depuis le $\mathrm{XXI}^{\mathrm{e}}$ siècle, le monde se trouve confronté à une crise aiguë: les changements climatiques (PNUD, 2007). Ce phénomène de changements climatiques est principalement lié aux émissions importantes de gaz à effet de serre (GES) d'origines naturelle et anthropique (GIEC, 2007). Selon le PNUD (2007), ces aléas climatiques sont les plus importants et les plus urgents de tous les problèmes environnementaux. Les connaissances actuelles sur les effets du changement climatique montrent que les domaines impactés sont divers et les projections d'impacts futurs sont très alarmantes aussi bien dans le secteur des ressources d'eau douce et leur gestion, des écosystèmes, de la nourriture, des fibres et produits forestiers, des systèmes côtiers et régions de basses-terres que dans le secteur de la santé (GIEC, 2007). L'Afrique en général et l'Afrique de l'Ouest en particulier sont plus 
vulnérables aux changements climatiques à cause de certaines de leurs caractéristiques physiques et socio-économiques qui les prédisposent à être affectées, de façon disproportionnée, par les effets négatifs des variations du climat (CRDI et IDID, 2007; GIEC, 2007). De même, l'Organisation Mondiale de la Météorologie (OMM) et le Programme des Nations Unies pour l'Environnement (PNUE) (2002) prédisent que ces changements auront des répercussions socio-économiques et environnementales négatives principalement en Afrique subsaharienne.

Les sols de cette partie de l'Afrique sont propices au développement et à l'activité de certains êtres vivants contribuant aux émissions mondiales de GES. Au nombre de ces êtres, figurent les termites. En effet, ces insectes sont très répandus dans les zones tropicales où la famille des Termitidae occupe plus de 2/3 des terres et leur activité contribue au recyclage de la matière organique végétale et à l'humification de l'écosystème terrestre (Lefebvre, 2008). Compte tenu de leur forte densité, surtout en régions tropicales, les termites contribuent significativement à l'émission de traces de GES comme le $\mathrm{CO}_{2}$ et le $\mathrm{CH}_{4}$. Zimmerman et al. (1982) ont évalué la contribution des termites à l'émission globale de $\mathrm{CH}_{4}$ à plus de 45\%; mais d'autres études ont noté une contribution mondiale de $4 \%$ de $\mathrm{CH}_{4}$ et $2-11,3 \%$ de $\mathrm{CO}_{2}$ en se basant sur les différences inter-espèce de termites (Sanderson, 1996; Konaté et al., 2003). Une étude plus récente a montré que les termitières de Cubitermes fungifaber contribuent à hauteur de $0,15 \%$ aux émissions mondiales de $\mathrm{CH}_{4}$ et d'importantes quantités de $\mathrm{CO}_{2}$ dans la savane sud-soudanienne du Burkina Faso (Brümmer et al., 2009). Il est établi que les émissions de $\mathrm{CO}_{2}$ et $\mathrm{CH}_{4}$ sont fonction des paramètres environnementaux et de la zone écologique (Korb et Linsenmair, 2000; Jamali et al., 2009). Toutefois, très peu d'études ont porté sur l'émission de ces GES par d'autres espèces de termites connues que regorge ce pays.

La présente étude a pour objectif de quantifier les émissions de $\mathrm{CO}_{2}$ et $\mathrm{CH}_{4}$ à partir des termitières de Macrotermes bellicosus dans la région du centre au Burkina Faso.

\section{MATERIEL ET METHODES}

\section{Site expérimental}

Le site d'étude est localisé dans la réserve de Somgandé $\left(12^{\circ} 24^{\prime} \mathrm{N}, 1^{\circ} 29^{\prime} \mathrm{O}\right.$, altitude $294 \mathrm{~m}$ ) dans la ville de Ouagadougou. Elle s'étend sur une superficie de 15 ha où des activités agricoles sont réalisées en saison pluvieuse. L'étude de l'évaluation des émissions de $\mathrm{CO}_{2}$ et de $\mathrm{CH}_{4}$ a été faite entre mai et juin 2010 sur deux différentes termitières de la même espèce de termites Macrotermes bellicosus.

\section{Mesure des concentrations de $\mathrm{CO}_{2}$ et $\mathrm{CH}_{4}$ Implantation des tubes de piégeage de gaz}

La capture des gaz émis au niveau de chaque termitière de $M$. bellicosus a été effectuée par trois petits trous creusés dans la muraille des termitières au niveau des galeries d'exoécies (perforations). De petits tubes ont été ensuite introduits dans chaque trou, puis un tube en PVC d'environ $1 \mathrm{~L}$ ( $8 \mathrm{~cm}$ de diamètre interne et $21 \mathrm{~cm}$ de long) sectionné à la base a été ajusté au trou et enfoncé en quasi-totalité dans la termitière de manière à recueillir les gaz. L'extrémité supérieure du tube a été munie d'un bouchon septum permettant l'échantillonnage des gaz au moyen d'une seringue étanche graduée de 10 $\mathrm{ml}$. Sur chaque termitière étudiée, trois de ces pièges à gaz ont été disposés dans différents compartiments et gardés dans cette position durant l'étude (Figure 1).

\section{Température interne des termitières}

La température interne de chaque termitière a été mesurée, à proximité de chaque tube de piégeage, par un thermomètre à sonde longue de $10 \mathrm{~cm}$ (Bioblock Scientific Minitherm) lors de chaque échantillonnage. 


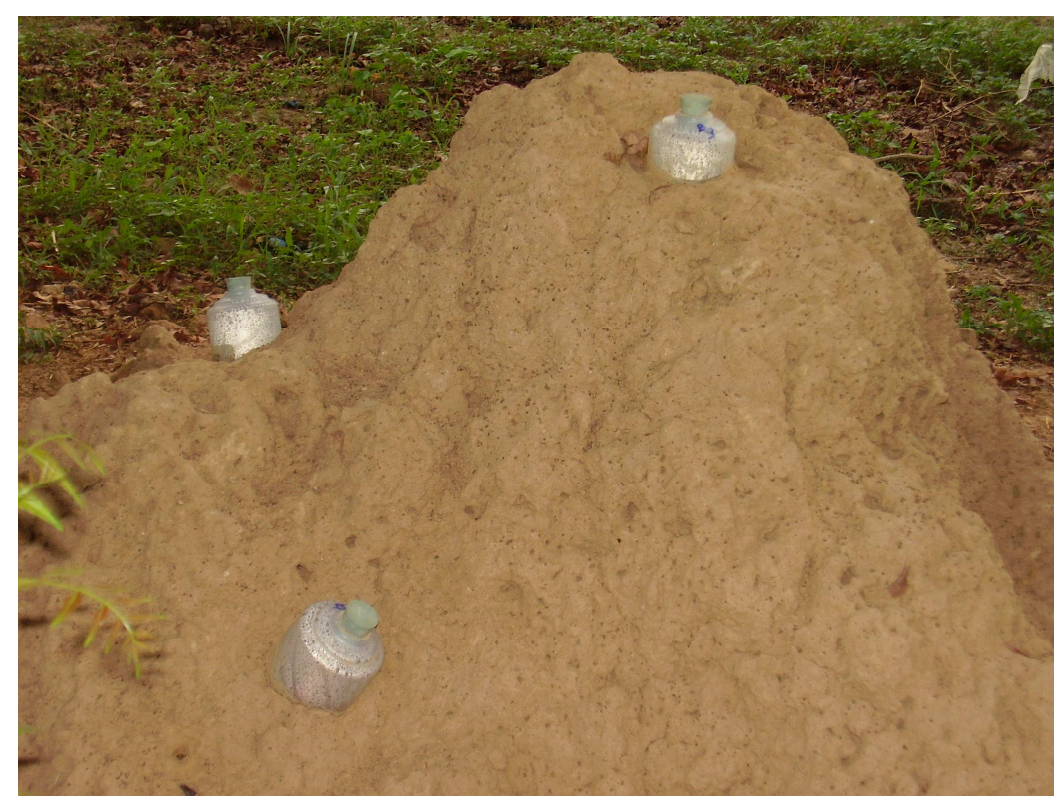

Figure 1: Dispositifs de piégeage de gaz sur une termitière de M. bellicosus.

Bien avant la prise de température, un clou (longueur $50 \mathrm{~cm}$ et diamètre $0,7 \mathrm{~cm}$ ) a été enfoncé dans la termitière à partir d'une exoécie afin d'obtenir un petit trou pour l'introduction de la sonde. La température du milieu ambiant a été aussi relevée à chaque prélèvement gazeux.

\section{Échantillonnage gazeux et mesure des quantités de $\mathrm{CO}_{2}$ et $\mathrm{CH}_{4}$}

L'émission de $\mathrm{CO}_{2}$ sur chaque termitière a été suivie chaque $12 \mathrm{~h}$ (de $6 \mathrm{~h}$ à 18 $\mathrm{h}$ pendant la journée et de $18 \mathrm{~h}$ à $6 \mathrm{~h}$ la nuit) durant 3 jours consécutifs, et celle de $\mathrm{CH}_{4}$, tous les 5 jours pendant 30 jours (en raison de la faible détection du méthane) pour évaluer les quantités émises de ces gaz. $4 \mathrm{ml}$ d'échantillon gazeux ont été prélevés de l'espace gazeux des tubes en PVC au moyen de seringues graduées $(10 \mathrm{ml})$ étanches et stockés dans $4 \mathrm{ml}$ de tube Venoject@ sec. Au total, 18 et 21 échantillons pour le dosage respectif de $\mathrm{CO}_{2}$ et de $\mathrm{CH}_{4}$ ont été prélevés de chaque termitière. Les échantillons ont été rapportés immédiatement au laboratoire pour être analysés par chromatographie en phase gazeuse sur le Girdel série 30 muni d'un détecteur à conductivité thermique (TCD) et couplé à un enregistreur potentiométrique SERVOTRACE type Sefram Paris de $1 \mathrm{mV}$.

Le chromatographe a été équipé de deux colonnes remplies Porapak Q80/100 (pour doser le $\mathrm{CH}_{4}$ ) et Porapak Q100/120 (pour doser le $\mathrm{CO}_{2}$ ) montées en parallèle et programmé comme suit: température de l'injecteur $90{ }^{\circ} \mathrm{C}$, température du four $60^{\circ} \mathrm{C}$, température du détecteur $100{ }^{\circ} \mathrm{C}$, pressions du gaz vecteur hydrogène 1 bar (pour doser le $\mathrm{CO}_{2}$ ) et 2 bars (pour doser le $\mathrm{CH}_{4}$ ), courant filament $150 \mathrm{~mA}$ et atténuation $4.1 \mathrm{ml}$ de chaque échantillon gazeux a été prélevé des tubes Venoject@ de $4 \mathrm{ml}$ puis injecté à laide d'une seringue étanche graduée de $1 \mathrm{ml}$ dans le chromatographe.

\section{Courbes d'étalonnage de $\mathrm{CO}_{2}$ et $\mathrm{CH}_{4}$}

A partir du témoin $\mathrm{CO}_{2}$ pur (pureté $99 \%$ ), des volumes de $10 ; 15 ; 20 ; 25$ et $30 \mu 1$ ont été injectés dans le Girdel. Les réponses obtenues ont permis d'établir l'équation suivante de la courbe de régression: Volume 
$\mathrm{CO}_{2}(\mu \mathrm{l})=21,574 \times$ Aire du pic $\mathrm{CO}_{2}-0,0182$ $\left(r^{2}=0,9994\right)$.

Le $\mathrm{CH}_{4}$ étalon a été synthétisé avec une pureté de $90 \%$. Comme précédemment, différents volumes de $\mathrm{CH}_{4}(50 ; 100 ; 150$ et $200 \mu \mathrm{l})$ ont été injectés afin d'obtenir l'équation de la courbe de régression: Volume $\mathrm{CH}_{4}(\mu \mathrm{l})=29,68 \times$ Aire pic $\mathrm{CH}_{4}+0,0018$ $\left(r^{2}=0.9993\right)$.

\section{Analyse statistique}

SPSS statistics 17.0 a été utilisé pour l'analyse statistique des données. L'ANOVA à 1 facteur a été réalisée pour identifier les différences significatives des valeurs moyennes de $\mathrm{CO}_{2}$, et de température pour chaque termitière et entre les termitières $(n=2)$ selon les périodes diurne et nocturne. Le Least Standardized Difference (LSD) test a permis la détermination des moyennes significativement différentes $(\mathrm{p}<0,05)$ suivant les variables considérées. Pour étudier la relation entre les valeurs de $\mathrm{CO}_{2}$ et de température de chaque termitière, la corrélation de Pearson a été utilisée au seuil de 0.05 le jour et la nuit séparément.

\section{RESULTATS}

\section{Émissions de $\mathrm{CO}_{2}$ et $\mathrm{CH}_{4}$ au niveau des termitières}

Les analyses ont révélé des concentrations de $\mathrm{CO}_{2}$ variables selon le cycle journalier (Figure 2). En effet, sur chaque termitière, les concentrations de $\mathrm{CO}_{2}$ ont été plus importantes pendant la nuit que le jour sans aucune différence significative (Termitière $\mathrm{T}_{1}, \mathrm{p}=0,809$; Termitière $\mathrm{T}_{2}, p=$ 0,385). 22368 parties par million (ppm) de $\mathrm{CO}_{2}$ en moyenne ont été enregistrées la nuit contre 21397 ppm le jour dans $\mathrm{T}_{1}$, et 12804 ppm la nuit contre $9985 \mathrm{ppm}$ le jour dans $\mathrm{T}_{2}$. Par ailleurs, les émissions moyennes de $\mathrm{CO}_{2}$ ont été significativement différentes entre les termitières $(\mathrm{p}=0,004)$ aussi bien pendant le jour $(\mathrm{p}=0,008)$ que la nuit $(\mathrm{p}=0,035)$. De plus, les concentrations de $\mathrm{CO}_{2}$ de $\mathrm{T}_{1}$ ont été deux fois supérieures à celles de $\mathrm{T}_{2}$. Bien que ces termitières appartiennent à la même espèce de termite, elles présentent chacune une architecture différente qui peut jouer un rôle dans le flux d'émission de gaz (Figures 3, 4).

Les températures ambiantes et celles des termitières ont été, en général, plus élevées le jour que la nuit (Figure 2). Le jour, les températures moyennes ont été de l'ordre de $33,3 \pm 1,5^{\circ} \mathrm{C}, 32,1 \pm 1,7^{\circ} \mathrm{C}$ et $29,4 \pm 0,5$ ${ }^{\circ} \mathrm{C}$ respectivement dans le milieu ambiant et pour les termitières $T_{1}$ et $T_{2}$. Pendant la nuit, elles ont été de $29,8 \pm 1,7{ }^{\circ} \mathrm{C}, 27,7 \pm 0,5^{\circ} \mathrm{C}$ et $26,2 \pm 0,7{ }^{\circ} \mathrm{C}$ respectivement pour les termitières $T_{1}, T_{2}$ et le milieu ambiant. Les températures internes moyennes entre le jour et la nuit ont été significativement différentes dans $\mathrm{T}_{1}(\mathrm{p}<0,05)$ contrairement dans $\mathrm{T}_{2}$. Toutefois, il faut noter que les températures ambiantes ont été supérieures à celles des termitières durant le jour à l'opposé de la nuit (Figure 2). De façon explicite, le gradient de température entre l'intérieur des termitières et le milieu ambiant (extérieur) a été croissant le jour et décroissant la nuit. Les valeurs moyennes de $\mathrm{CO}_{2}$ et de température chez $\mathrm{T}_{1}$ ont présenté une faible corrélation négative aussi bien le jour $(\mathrm{r}=-0,067)$ que la nuit $(\mathrm{r}=$ - 0,355). Dans $T_{2}$, elles ont été faiblement corrélées le jour $(\mathrm{r}=-0,390)$ contrairement la nuit $(r=0,675 ; p=0,023)$.

La présence de $\mathrm{CH}_{4}$ a été révélée également mais à de très faibles quantités (< $0,5 \mathrm{ppm}$ ou $<0,02 \mu \mathrm{mol}$ par jour) les 14 premiers jours (du 18 au 31 mai). Mais audelà de cette période, nous avons observé une légère augmentation des pics de $\mathrm{CH}_{4}$ équivalent à 0,5 ppm/jour. L'ensemble de tous ces résultats pourrait découler de divers aspects tels que les conditions intrinsèques des termitières en lien avec le milieu écologique que nous comptons discuter. 


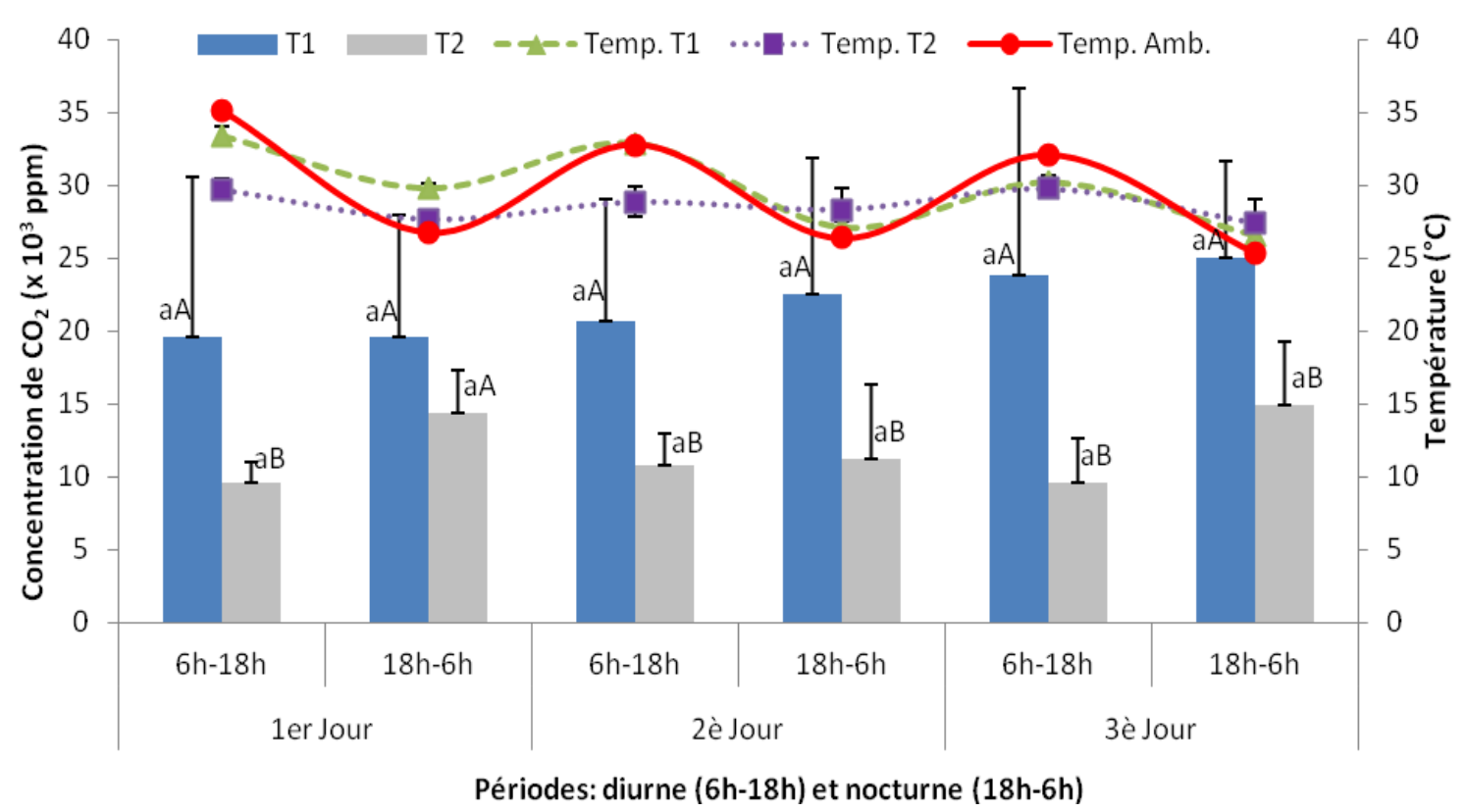

Figure 2: Concentrations de $\mathrm{CO}_{2}(n=3, \pm \mathrm{SE})$ mesurées des termitières $\mathrm{T}_{1}$ et $\mathrm{T}_{2}$ de $M$. bellicosus selon les périodes diurne ( $6 \mathrm{~h}$ à $18 \mathrm{~h})$ et nocturne $(18 \mathrm{~h}$ à $6 \mathrm{~h})$ et la variation de températures internes $(n=3, \pm \mathrm{SE})$ des termitières et du milieu ambiant.

Les histogrammes représentent les concentrations de $\mathrm{CO} 2$ des termitières, les courbes indiquent les températures des termitières (Temp. T1 et Temp T2) et du milieu ambiant (Temp. Amb.). Les lettres majuscules indiquent les différences significatives $(p=0,004)$ entre les termitières dans chaque période. Les lettres minuscules montrent les différences non significatives pour chaque termitière ( $\mathrm{p}=0,809$ et 0,385 pour $\mathrm{T} 1$ et $\mathrm{T} 2$, respectivement) entre les périodes diurnes et nocturnes.

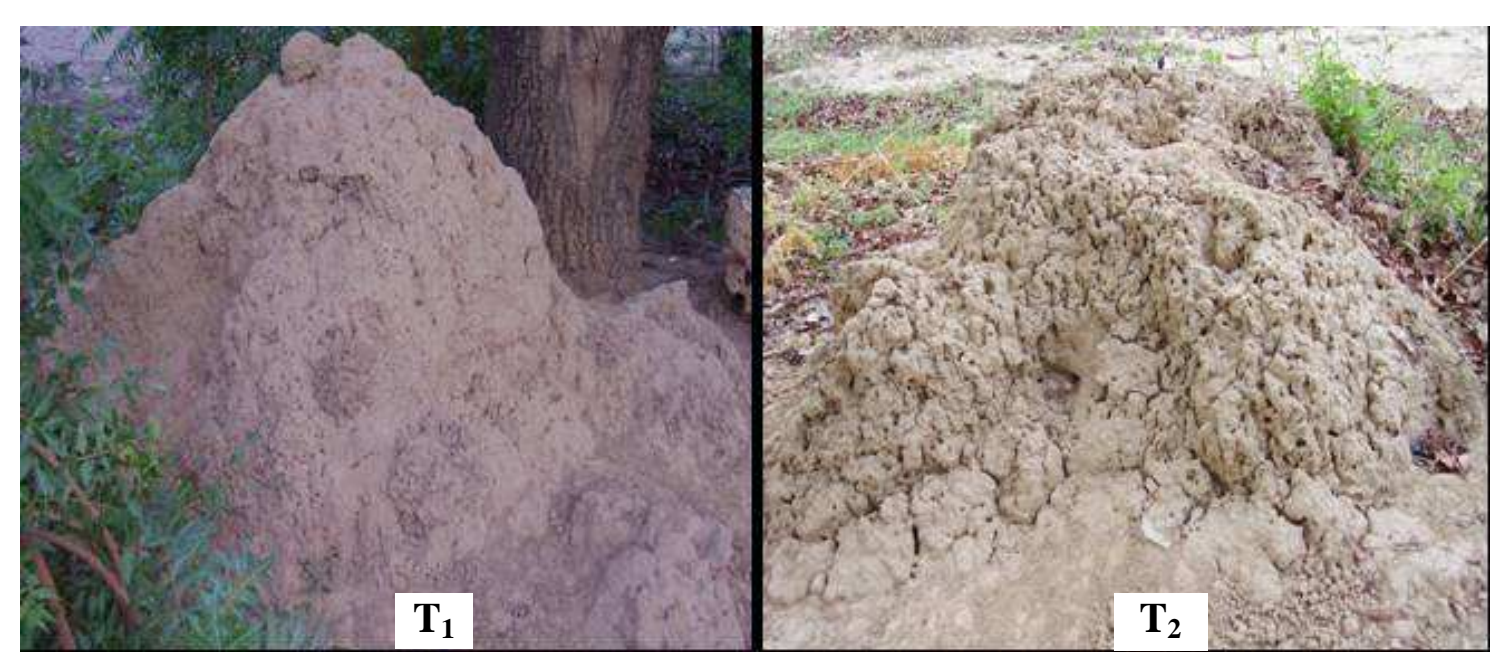

Figure 3: Structure des termitières de Macrotermes bellicocus de l'étude. 


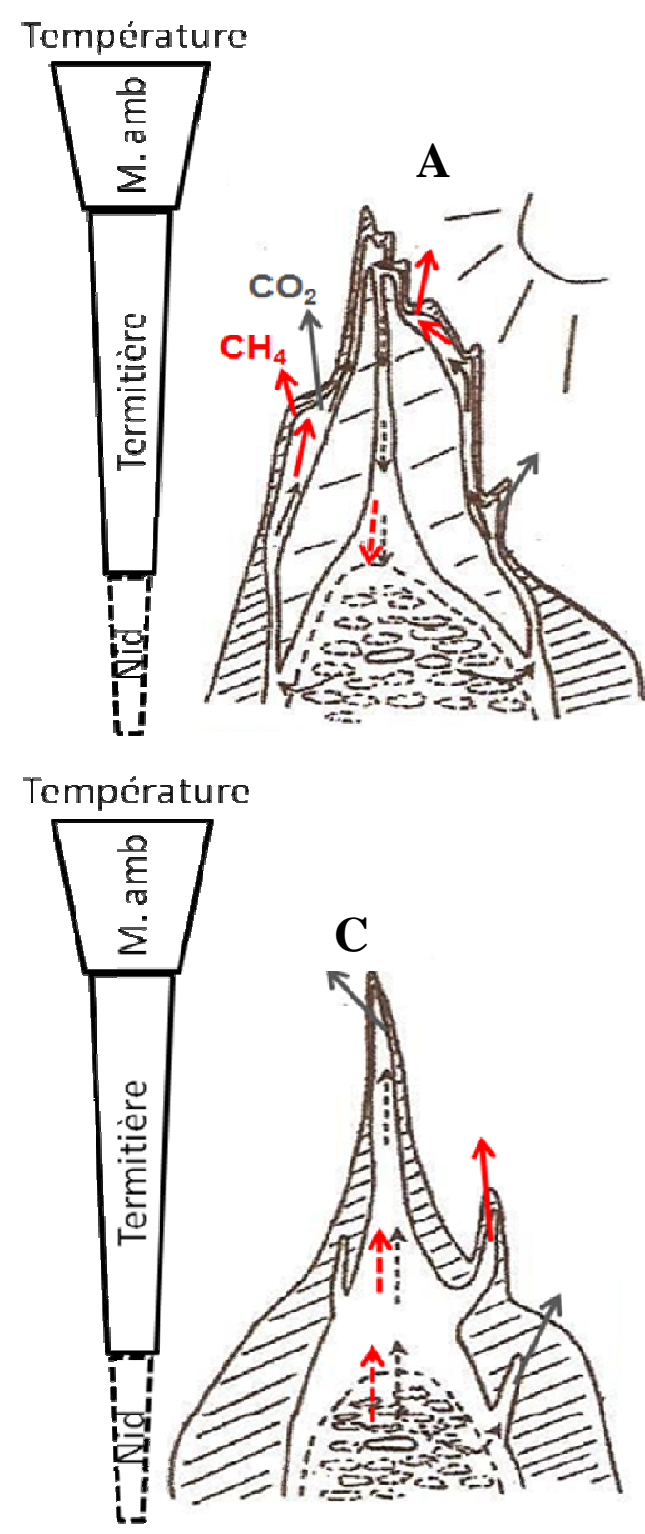

\section{Tempćrature}

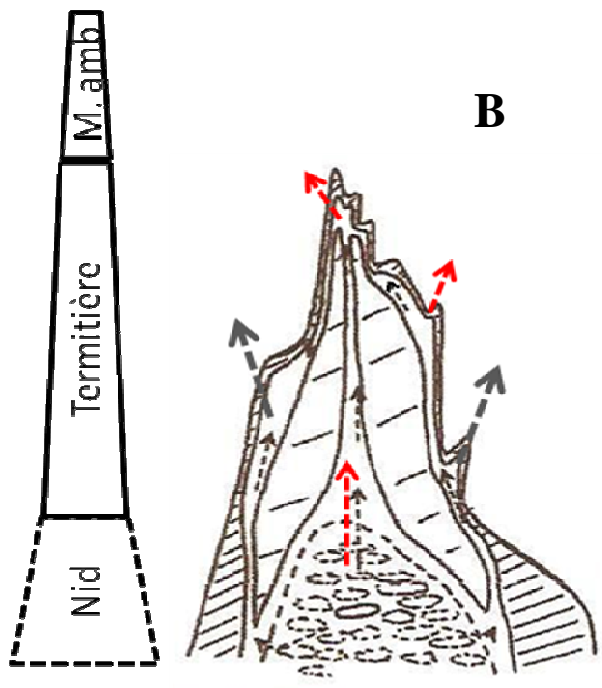

\section{Température}

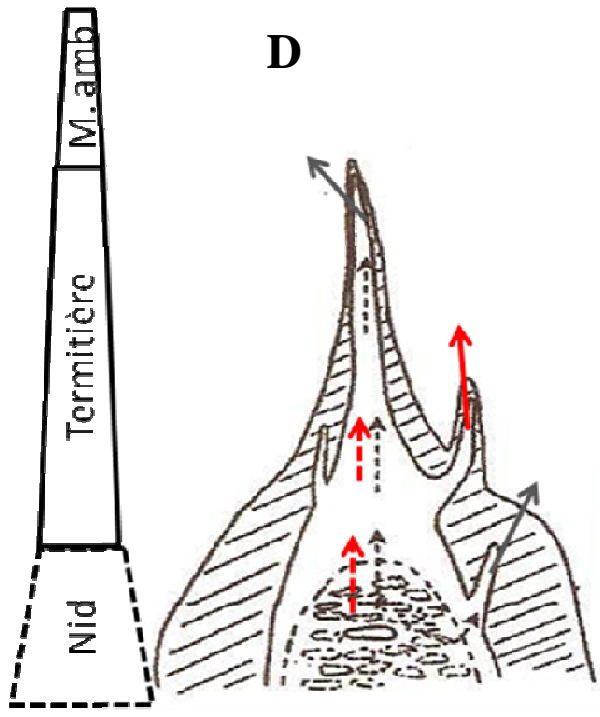

Figure 4: Mécanismes de ventilation supposés dans les termitières de Macrotermes bellicosus adaptés de Korb et Linsenmair (2000).

(A) Termitière cathédrale, le jour: flux bidirectionnel des gaz avec faible courant gazeux (flèches pleines) en périphérie et courant élevé (flèches en tiret) dans le canal central. (B) Termitière cathédrale, la nuit: flux unidirectionnel des gaz avec important courant dans le canal central et en périphérie. (C) et (D) Termitière dôme, respectivement le jour et la nuit: flux unidirectionnel des gaz avec important courant central et faible courant en périphérie. Les cadres à côté des termitières montrent les températures: températures mesurées du milieu ambiant (M. amb.) et de la termitière (cadre plein), températures supposées dans le nid de la termitière (cadre en tiret). 


\section{DISCUSSION}

Les concentrations de $\mathrm{CO}_{2}$, dès le premier jour, ont été légèrement plus élevées le jour que la nuit dans la termitière $\mathrm{T}_{1}$. Cela pourrait être lié d'une part à l'activité observée de nombreux termites ouvriers qui ont eu tendance à réparer les différents compartiments endommagés et à la présence de soldats alertés quelque temps après l'installation des dispositifs de piégeage d'autre part. Mais dans l'ensemble, les résultats obtenus sont en adéquation avec ceux de Korb et Linsenmair (1999) qui ont également rapporté une concentration de $\mathrm{CO}_{2}$ supérieure la nuit (13400 ppm) à celle du jour (11200 ppm) au niveau des termitières de $M$. bellicosus dans la savane guinéenne de Côte d'Ivoire. Par contre, Darlington et al. (1997) indiquent que l'espèce $M$. jeanneli produit plus de $\mathrm{CO}_{2}$ le jour $(6000 \mathrm{ppm})$ que la nuit (3000 ppm) sur des intervalles de $3 \mathrm{~h}$ pendant 24 h. En extrapolant nos résultats sur ce même intervalle de temps, nous enregistrons des concentrations de $\mathrm{CO}_{2}$ allant de $2400 \mathrm{ppm}$ le jour à 6258 ppm la nuit d'où comparables à celles de ces derniers auteurs. Cette augmentation de concentration de $\mathrm{CO}_{2}$ avec la température interne pendant la nuit au niveau des termitières, en l'occurrence $T_{2}$, pourrait être la résultante des mécanismes de ventilation en accord avec Korb et Linsenmair (2000) résumés dans la Figure 4 et également de l'activité intense des termites pendant cette même période (observation sur le terrain). Durant le jour, les termites se trouvent quasiment concentrés dans leur nid et les exoécies de la muraille sont scellées. Pendant cette période, la chaleur ambiante augmente la température de la périphérie de la termitière, entraînant ainsi la diffusion de gaz à l'intérieur du nid par le tunnel central sous l'effet d'une forte convection du courant d'air (Figure 4A). Par contre, durant la nuit, les termites ouvriers et soldats ouvrent les exoécies préalablement scellées pour mener leur activité de récolte hors du nid et pour sécuriser la périphérie de la termitière; la température du nid serait supérieure à celle de la périphérie de la termitière, ce qui permettrait une importante sortie de gaz du nid à travers le tunnel central et les canaux périphériques ouverts de la termitière sous l'effet de forte convection du courant d'air chaud (Figure 4B) (Korb et Linsenmair, 1999, 2000). Ces deux mécanismes distincts expliqueraient la différence relativement importante des quantités de $\mathrm{CO}_{2}$ dans la termitière $T_{2}$ entre le jour et la nuit, bien qu'elles ne soient pas significativement différentes. Quant à la termitière $\mathrm{T}_{1}$, cette différence est faible du fait qu'il existerait un seul type de mécanisme de ventilation notamment celui conduisant un faible flux de gaz vers l'extérieur de la termitière corrélé à la compacité de sa muraille réduisant donc les échanges gazeux (Figures 4C, D).

La température à l'intérieur du nid que nous n'avons pas pu déterminer pourrait avoir une influence directe sur le métabolisme des termites eux-mêmes dans la production plus ou moins importante de $\mathrm{CO}_{2}$. Les termites tendent à garder la température de leur nid constante $\left(\begin{array}{llll}25 & \text { à } & 30 & { }^{\circ} \mathrm{C}\end{array}\right)$ à travers divers mécanismes face aux variations climatiques externes pour assurer leur métabolisme et celui des champignons du genre Termitomyces cultivés (Jones et Oldroyd, 2007; Zaremski et al., 2009). Ces champignons, bien qu'ils servent à la dégradation de la meule (aliment de base pour les termites champignonnistes), participent davantage à la production de $\mathrm{CO}_{2}$ (Konaté et al., 2003; Gomathi et al., 2009; Sawadogo et al., 2011).

De plus, la différence significative de concentrations de $\mathrm{CO}_{2}$ entre les deux termitières $(\mathrm{p}=0,04)$ dépendrait du mode de construction des termitières elles-mêmes. En effet, la termitière $T_{1}$ a une architecture plus compacte avec une muraille plus épaisse et compte moins d'exoécies à petit diamètre qui réduiraient la déperdition de gaz et de chaleur interne contrairement à la termitière $\mathrm{T}_{2}$ 
(Figure 3). De ce fait, Korb et Linsenmair (1998, 1999) et Korb (2007) ont évoqué l'influence de l'épaisseur de la muraille des termitières où la concentration de $\mathrm{CO}_{2}$ a été plus accrue au niveau des termitières en « dôme » des forêts que celles en « cathédrale » des savanes. Les termitières en «cathédrale » sont donc reconnues comme ayant un système de ventilation et d'échange gazeux plus développé. Nous pouvons en déduire que plus la muraille d'une termitière est épaisse avec peu de perforations, moins il y a perte de chaleur dans la termitière (par exemple la température élevée dans la termitière $T_{1}$ comparativement à $\mathrm{T}_{2}$ ) et moins il $\mathrm{y}$ a émission de gaz hors de la termitière.

La zone écologique pourrait avoir un impact sur le type d'architecture des termitières avec pour corolaire le système de ventilation, le nombre de termites, la taille des termitières (Josens et Soki, 2010) et surtout l'émission de $\mathrm{CO}_{2}$. Darlington et al. (1997) et Korb et Linsenmair (1999) ont mesuré respectivement moins de 20000 ppm et 6000 ppm de $\mathrm{CO}_{2}$ en savane et/ou en forêt près des sources humides.

Quant au méthane, de faibles quantités de ce gaz ont été décelées des deux termitières au cours de l'étude. Seiler et al. (1984), Khalil et al. (1990) et Delmas et al. (1992) ont déduit que la quasi-totalité du méthane produit du nid des termites est oxydée par les bactéries du sol. Cela a été aussi justifié par la consommation du méthane élaboré par les termites incubés avec leur propre sol lors des travaux de Sawadogo et al. (2011). En fait, durant les 14 premiers jours de l'expérimentation, le sol a présenté un état exondé et cet état favoriserait la consommation du méthane par les bactéries méthanotrophes selon Roger et Le Mer (2003). L'observation d'une légère augmentation du pic de méthane après 14 jours pourrait s'expliquer par: (1) l'accumulation de méthane produit en faible quantité serait graduelle et s'effectuerait surtout en fin d'étape au cours du processus de méthanisation lent, et (2) l'humidification de la zone par la pluie, à partir de fin mai, accentuerait la production du $\mathrm{CH}_{4}$.

Dans le dernier cas, Korb et Linsenmair (1999), Brümmer et al. (2009), ainsi que Jamali et al. (2009, 2011) ont montré que les variations saisonnières ont un impact sur les termitières dans l'émission de gaz et que, de ce fait, la saison humide serait la plus propice aux émissions de dioxyde de carbone et surtout de méthane car l'activité des méthanotrophes est amoindrie par le manque d'oxygène dans un milieu inondé (Roger et Le Mer, 2003; Dianou, 2005). Le sol des termitières serait un puits de méthane (Sugimoto et al., 1998) qui abriterait donc des bactéries méthanotrophes.

\section{Conclusion}

Les termites développent des stratégies de ventilation selon la structure de la termitière pour la thermorégulation au sein de leur habitat en fonction de la température du milieu extérieur. Ce système favorise ainsi le flux de gaz produits par les termites en suivant le gradient de température inverse entre le nid, la termitière et l'atmosphère selon le cycle journalier. A cela s'ajoute l'activité des termites pour modifier la structure de leur termitière selon le cycle diurne ou nocturne. L'éventuelle existence des bactéries méthanotrophes dans le sol des termitières pourrait contribuer donc à la réduction de méthane. Cette dernière, couplée au mécanisme de ventilation des gaz, semblerait être efficace pour l'adaptation des termites dans leur zone écologique et aux conditions de l'environnement. Ainsi, le suivi de la production des gaz durant les différentes saisons et les variations de température seraient nécessaires pour apprécier l'incidence de ces facteurs sur les quantités globales de $\mathrm{CO}_{2}$ et $\mathrm{CH}_{4}$ produites au niveau des termitières de ladite espèce de notre zone d'étude. 


\section{REMERCIEMENTS}

Nous

remercions

Adama

OUEDRAOGO pour son accord à l'accès au site expérimental, Adama OULLIE du Lycée Marien N'Gouabi (Ouagadougou) pour nous avoir synthétisé du méthane. Nous remercions Pr Aboubakar S. OUATTARA, Pr Nicolas BARRO, Dr Aly SAVADOGO pour leurs contributions et également les structures financières du CRSBAN (UEMOA) et le CIOSPB pour leur appui dans la conduite des travaux de recherche.

\section{REFERENCES}

Brümmer C, Papen H, Wassmann R, Brüggermann N. 2009. Flux of $\mathrm{CH}_{4}$ and $\mathrm{CO}_{2}$ from soil and termite mounds in south Sudanian savanna of Burkina Faso (West Africa). Global Biogeochemical Cycles, 23, GB1001, doi:10.1029/ 2008 GB003237.

CRDI, IDID. 2007. Projet de renforcement des capacités d'adaptation des acteurs ruraux béninois face aux changements climatiques. ACCA.

Darlington JPEC, Zimmerman PR, Greenberg J, Westeberg C, Bakwin P. 1997. Production of metabolic gases by nests of the termite Macrotermes jeanneli in Kenya. Journal of Tropical Ecology, 13: 491-510.

Delmas RA, Servant J, Tathy JP, Cros B, Labat M. 1992. Sources and sinks of methane and carbon dioxide exchanges in mountain forest in equatorial Africa. Journal of Geophysical Research, 97: 6169-6179.

Dianou D. 2005. Étude des bactéries sulfatoréductrices, méthanotrophes et les archaebactéries méthanogènes dans les sols de rizière: influence des interactions sur la production du riz et sur l'émission du méthane dans l'atmosphère. Thèse de doctorat, Université de Ouagadougou, Ouagadougou, p. 243.
GIEC. 2007. Bilan 2007 des changements climatiques. Contribution des Groupes de travail I, II et III au quatrième rapport d'évaluation du groupe d'experts intergouvernemental sur l'évolution du climat. [Équipe de rédaction principale, Pachauri RK et Reisinger A]. GEIC, Genève, Suisse, p. 103.

Gomathi V, Ramasamy K, Reddy MRVP, Ramalaksmi A, Ramanathan A. 2009. Methan emission by gut symbionts of termites. Acad. J. Plant Sci., 2(3): 189194.

Jamali H, Livesely S, Arndt S. 2009. Methane fluxes from the mound-building termites in the tropical savannas of Australia. The University of Melbourne, Department of Forest and Ecosystem Science, p.15.

Jamali H, Livesley SJ, Dawes TZ, Cook GD, Hutley LB, Arndt SK. 2011. Diurnal and seasonal variations in $\mathrm{CH}_{4}$ flux from termite mounds in tropical savannas of the Northern Territory, Australia. Journal Agricultural and Forest Meteorology, 151 : 1471-1479.

Jones JC, Oldroyd BP. 2007. Nest Thermoregulation in Social Insects. Advances in Insect Physiology, 33: 153192.

Josens G, Soki K. 2010. Relation between termite numbers and the size of their mounds. Insect. Soc., DOI 10.1007/s00040-010-0085-2.

Khalil MAK, Rasmussen RA, French JRJ, Holt JA. 1990. The influence of termites on atmospheric trace gases: $\mathrm{CH}_{4}, \mathrm{CO}_{2}$, $\mathrm{CHCl}_{3}, \quad \mathrm{~N}_{2} \mathrm{O}, \mathrm{CO}, \mathrm{H}_{2}$ and light hydrocarbons. Journal of Geophysical Research, 95: 3619-3634.

Konaté S, Le Roux X, Verdier B, Lepage M. 2003. Effect of underground fungusgrowing termites on carbon dioxide emission at the point- and landscapescales in an African savanna. Functional Ecology, 17: 305-314. 
Korb J, Linsenmair KE. 1998. The effect of temperature on the architecture and distribution of Macrotermes bellicosus (Isoptera, Macrotermitinae) mounds in different habitats of a West of Africa Guinnea savanna. Insect Soc., 44: 51-65.

Korb J, linsenmair KE. 1999. The architecture of termite mounds: a result of a trade-off between thermoregulation and gas exchange. Behavioral Ecology, 10: 312316.

Korb J, Linsenmair KE. 2000. Ventilation of termite mound: new results require new model. Behavioral Ecology, 11: 486494.

Korb J. 2007. Termites. Current Biology, 17(23): 995-999.

Lefebvre T. 2008. Associations biologiques entre les termites du genre Nasutitermes et leur microflore actinomycétale: spécificité et évolution. Thèse de Doctorat, Université Paris Est, Paris, p. 168.

OMM, PNUE. 2002. Bilan des changements climatiques 2001. Rapport de synthèse, $p$ 204.

PNUD. 2007. Rapport Mondial sur le Développement Humain 2007/2008: La Lutte contre le Changement Climatique: un Impératif de Solidarité Humaine dans un Monde Divisé: Oxford University Press: New York.

Roger P, Le Mer J. 2003. Les sols: sources et puits de méthane. Étude et Gestion des Sols, 10(4): 331- 345.
Sanderson MG. 1996. Biomass of termites and their emissions of methane and carbon dioxide: A global database. Global Biogeochem. Cycles, 10: 543557.

Sawadogo BJ, Dianou D, Traoré SA. 2011. Effects of temperature and termite' substrate on methane and carbon dioxide emissions from Macrotermes bellicosus and Microcerotermes dubius cultures. Middle-East J. Sci. Res., 9(1): 75-83.

Seiler W, Conrad R, Scharffe D. 1984. Field studies of methane emission from termites nests into the atmosphere and measurements of methane uptake by tropical soil. Journal of Atmospheric Chemistry, 1: 171-186.

Sugimoto A, Inoue $\mathrm{T}$, Kirtibutr $\mathrm{N}$, Abe $\mathrm{T}$. 1998. Methane oxidation by termite mounds estimated by the carbon isotopic composition of methane. Global Biogeochemical Cycles, 12(4): 595-605.

Zaremski A, Fouquet D, Louppe D. 2009. Les Termites dans le Monde (ed. Quæ). Collection Guide Pratique: Versailles.

Zimmerman PR, Greenberg JP, Wandiga SO, Crutzen PJ. 1982. Termite: a potentially large source of atmospheric methane, carbon dioxide and molecular hydrogen. Science, 218(5): 563-565. 